\title{
Chemical potentials and parity breaking: the Nambu-Jona-Lasinio model
}

\author{
A. A. Andrianov ${ }^{1,2}$, D. Espriu ${ }^{2}$, X. Planells ${ }^{2, a}$ \\ ${ }^{1}$ V. A. Fock Department of Theoretical Physics, Saint-Petersburg State University, 198504 St. Petersburg, Russia \\ ${ }^{2}$ Departament d'Estructura i Constituents de la Matèria and Institut de Ciències del Cosmos (ICCUB), Universitat de Barcelona, \\ Martí i Franquès 1, 08028 Barcelona, Spain
}

Received: 23 December 2013 / Accepted: 5 February 2014 / Published online: 19 February 2014

(C) The Author(s) 2014. This article is published with open access at Springerlink.com

\begin{abstract}
We consider the 'two flavour' Nambu-JonaLasinio model in the presence of a vector and an axial external chemical potential and study the phase structure of the model at zero temperature. The Nambu-Jona-Lasinio model is often used as a toy replica of QCD and it is therefore interesting to explore the consequences of adding external vector and axial chemical potentials in this model, mostly motivated by claims that such external drivers could trigger a phase where parity could be broken in QCD. We are also motivated by some lattice analysis that attempt to understand the nature of the so-called Aoki phase using this simplified model. Analogies and differences with the expected behaviour in QCD are discussed and the limitations of the model are pointed out.
\end{abstract}

\section{Motivation}

In the last years, the possibility that parity breaks in QCD at high temperatures and/or densities has received a lot of attention [1-8]. Although parity is well known to be a symmetry of strong interactions, there are reasons to believe that it may be broken under extreme conditions. On the one hand, theoretical work using effective meson Lagrangians satisfying the QCD symmetries at low energies suggest that for some values of the vector chemical potential $\mu$ a new phase with an isotriplet pseudoscalar condensate may arise $[7,8]$. On the other hand, thermal fluctuations in a finite volume may lead to large topological fluctuations that induce a non-trivial axial quark charge that could be described in a quasi-equilibrium situation by an axial chemical potential $\mu_{5}$ [1-6,9-13].

Checking these claims in QCD is unfortunately very difficult. For one thing, finite density numerical simulations in the lattice present serious difficulties [14-19]. A vector chemical potential in gauge theories like QCD cannot easily be

\footnotetext{
a e-mail: xumeu@icc.ub.edu
}

treated and therefore simpler models hopefully reproducing the main features of the theory may be useful. Needless to say, non-equilibrium effects are also notoriously difficult to study non-perturbatively. However, an axial chemical potential is tractable on the lattice $[20,21]$ and with other methods [22,23].

In the present paper we shall consider the Nambu-JonaLasinio model (NJL) [24-30], which shares interesting features with QCD such as the appearance of chiral symmetry breaking. In the NJL modelisation, QCD gluon interactions among fermions are assumed to be replaced by some effective four-fermion couplings. Confinement is absent in the NJL model, but global symmetries can be arranged to be identical in both theories.

However, NJL is definitely not QCD and the present work does not attempt to draw definite conclusions on the latter theory; just to point out possible phases requiring further analysis.

Previously some authors have studied the effect of a vector chemical potential $\mu$ with three flavours [31] in the NJL model, but the consequences of including both a vector and an axial chemical potentials have not been considered so far to our knowledge. In this work, we will incorporate both chemical potentials with the purpose of unraveling the landscape of different stable phases of the theory. It turns out that the inclusion of $\mu_{5}$ changes radically the phase structure of the model and shows that $\mu$ is not a key player in ushering a thermodynamically stable phase where parity is violated in the NJL model, but $\mu_{5}$ is.

This paper is organised as follows. In Sect. 2 the NJL Lagrangian with the incorporation of $\mu$ and $\mu_{5}$ will be introduced. We describe how an effective potential is extracted when one introduces some effective light meson states and integrates out the fermion degrees of freedom. In Sect. 3 we show the gap equations of the model and the conditions for their stability. After that, the different stable phases of this 
model are presented and discussed. We show in Sect. 4 that a phase with an isospin singlet pseudoscalar condensate in addition to a scalar condensate is possible. It turns out that the conditions for this phase to be stable and exhibit chiral symmetry breaking too are such that one gets an inverted mass spectrum with $m_{\pi}>m_{\eta_{q}}$ and $m_{\sigma}>m_{a_{0}}$, which is quite different from QCD. In Sect. 4 we also present the main results of this work with plots of the evolution of the scalar and pseudoscalar condensates together with the main features of the phase transition. Finally, Sect. 5 is devoted to a summary of our conclusions.

\section{NJL Lagrangian with $\mu$ and $\mu_{5}$}

The starting point of this work is the NJL Lagrangian where we incorporate a vector and an axial chemical potentials $\mu$ and $\mu_{5}$, respectively. For two flavours and $N$ colours, we have

$$
\begin{aligned}
\mathcal{L}= & \bar{\psi}\left(\not \partial+m-\mu \gamma_{0}-\mu_{5} \gamma_{0} \gamma_{5}\right) \psi-\frac{G_{1}}{N}\left[(\bar{\psi} \psi)^{2}\right. \\
& \left.+\left(\bar{\psi} i \gamma_{5} \vec{\tau} \psi\right)^{2}\right]-\frac{G_{2}}{N}\left[(\bar{\psi} \vec{\tau} \psi)^{2}+\left(\bar{\psi} i \gamma_{5} \psi\right)^{2}\right],
\end{aligned}
$$

with a full $U(2)_{L} \times U(2)_{R}$ chiral invariance in the case that $G_{1}=G_{2}$, while if these constants differ, the $U(1)_{A}$ symmetry breaks and only $S U(2)_{L} \times S U(2)_{R} \times U(1)_{V}$ remains. One may introduce two doublets of bosonic degrees of freedom $\{\sigma, \vec{\pi}\}$ and $\{\eta, \vec{a}\}$ by adding the following chiral invariant term:

$$
\Delta \mathcal{L}=\frac{N g_{1}^{2}}{4 G_{1}}\left(\sigma^{2}+\vec{\pi}^{2}\right)+\frac{N g_{2}^{2}}{4 G_{2}}\left(\eta^{2}+\vec{a}^{2}\right) .
$$

These would be identified with their namesake QCD states (actually $\eta_{q}$ and $\vec{a}_{0}$ for the last two). Euclidean conventions will be used throughout. We bosonise the model following the same procedure as in [26].

After shifting each bosonic field with the quark bilinear operator that carries the corresponding quantum numbers, the Lagrangian (1) may be rewritten as

$$
\begin{aligned}
\mathcal{L}= & \bar{\psi}\left[\not \partial+m-\mu \gamma_{0}-\mu_{5} \gamma_{0} \gamma_{5}+g_{1}\left(\sigma+i \gamma_{5} \vec{\tau} \vec{\pi}\right)+g_{2}\left(i \gamma_{5} \eta\right.\right. \\
& +\vec{\tau} \vec{a})] \psi+\frac{N g_{1}^{2}}{4 G_{1}}\left(\sigma^{2}+\vec{\pi}^{2}\right)+\frac{N g_{2}^{2}}{4 G_{2}}\left(\eta^{2}+\vec{a}^{2}\right)
\end{aligned}
$$

which shows a redundancy related to the coupling constants $g_{1,2}$ that appear attached to each doublet and it is eventually related to their wave function normalisation. Without further ado we will take $g_{1}=g_{2}=1$.

Integration of the fermions will produce a bosonic effective potential (or free energy) and will allow one to study the different phases of the model. We will work in the mean field approximation and accordingly neglect fluctuations.
The results will be exact in the large $N$ limit. We have

$V_{\text {eff }}=\frac{N}{4 G_{1}}\left(\sigma^{2}+\vec{\pi}^{2}\right)+\frac{N}{4 G_{2}}\left(\eta^{2}+\vec{a}^{2}\right)-\operatorname{Tr} \log \mathcal{M}\left(\mu, \mu_{5}\right)$,

where the trace is understood to be performed in the isospin and Dirac spaces in addition to a 4-momentum integration of the operator in the momentum space. Throughout this article we will assume that $\mu>0$, namely we consider a baryon (as opposed to antibaryon) finite density. The invariance under $C P$ of the action ensures that the free energy (4) only depends on the modulus of $\mu$.

We also define the fermion operator

$$
\begin{aligned}
\mathcal{M}\left(\mu, \mu_{5}\right)= & \not \partial+(M+\vec{\tau} \vec{a})-\mu \gamma_{0}-\mu_{5} \gamma_{0} \gamma_{5} \\
& +i \gamma_{5}(\vec{\tau} \vec{\pi}+\eta),
\end{aligned}
$$

with the introduction of a constituent quark mass $M \equiv m+\sigma$.

In Appendix A we show that the dependence on both vector and axial chemical potentials does not change the reality of the fermion determinant. However, its sign remains undetermined, and in order to ensure a positive determinant, we shall consider an even number of "colours" ${ }^{1} N$ so that one can safely assume

$$
\operatorname{det}\left[\mathcal{M}\left(\mu, \mu_{5}\right)\right]=\sqrt{\operatorname{det}\left[\mathcal{M}\left(\mu, \mu_{5}\right)\right]^{2}}
$$

and hence use the calculations in Appendix A. If we just retain the neutral components of the triplets, this determinant can be written in the following way:

$$
\begin{aligned}
& \log \operatorname{det} \mathcal{M}\left(\mu, \mu_{5}\right)=\operatorname{Tr} \log \mathcal{M}\left(\mu, \mu_{5}\right) \\
& =\frac{1}{8} \operatorname{Tr} \sum_{ \pm}\left\{\log \left[-\left(i k_{0}+\mu\right)^{2}+\left(|\vec{k}| \pm \mu_{5}\right)^{2}+M_{+}^{2}\right]\right. \\
& \left.\quad+\log \left[-\left(i k_{0}+\mu\right)^{2}+\left(|\vec{k}| \pm \mu_{5}\right)^{2}+M_{-}^{2}\right]\right\}
\end{aligned}
$$

where

$M_{ \pm}^{2} \equiv(M \pm a)^{2}+(\eta \pm \Pi)^{2}$ and

$\operatorname{Tr}(1)=8 N T \sum_{n} \int \frac{d^{3} \vec{k}}{(2 \pi)^{3}}\left[k_{0} \rightarrow \omega_{n}^{F}=\frac{(2 n+1) \pi}{\beta}\right]$.

From now on, when we refer to the neutral pion condensate, we will write $\Pi$. Note that, as explained in Appendix A, one is able to write the determinant as the trace of an operator that is the identity in flavour space in spite of the initial non-trivial flavour structure. This facilitates enormously the calculations.

\footnotetext{
1 The choice of an even number of colours, unlike QCD, is simply a technical restriction to ensure the fermion determinant to be positive definite.
} 
In the search for stable configurations in the potential (4) we will need the derivatives of the fermion determinant, which are basically given by the function $K_{1}$ that we define as

$$
4 N K_{1}=\operatorname{Tr} \sum_{ \pm} \frac{1}{\left(i k_{0}+\mu\right)^{2}-\left[\left(|\vec{k}| \pm \mu_{5}\right)^{2}+M^{2}\right]}
$$

which is clearly divergent in the UV. In this work, we will deal with the NJL model using dimensional regularisation (DR) and a 3-momentum cut-off $(\Lambda)$ both at zero temperature [32-35]. The function $K_{1}$ depending on the regulator can be written as follows:

$$
\begin{aligned}
& K_{1}^{\mathrm{DR}}\left(M, \mu, \mu_{5}\right)=\frac{1}{2 \pi^{2}}\left[\Theta ( \mu - M ) \left\{\mu \sqrt{\mu^{2}-M^{2}}\right.\right. \\
& \left.+\left(2 \mu_{5}^{2}-M^{2}\right) \log \left(\frac{\mu+\sqrt{\mu^{2}-M^{2}}}{M}\right)\right\} \\
& \left.-\frac{1}{2} M^{2}+\frac{1}{2}\left(M^{2}-2 \mu_{5}^{2}\right)\left(\frac{1}{\epsilon}-\gamma_{E}+2-\log \frac{M^{2}}{4 \pi \mu_{R}^{2}}\right)\right],
\end{aligned}
$$$$
K_{1}^{\Lambda}\left(M, \mu, \mu_{5}\right)=\frac{1}{2 \pi^{2}}\left[\Theta ( \mu - M ) \left\{\mu \sqrt{\mu^{2}-M^{2}}\right.\right.
$$$$
\left.+\left(2 \mu_{5}^{2}-M^{2}\right) \log \left(\frac{\mu+\sqrt{\mu^{2}-M^{2}}}{M}\right)\right\}
$$$$
\left.-\frac{1}{2} M^{2}+\frac{1}{2}\left(M^{2}-2 \mu_{5}^{2}\right) \log \frac{4 \Lambda^{2}}{M^{2}}-\Lambda^{2}\right] \text {. }
$$

The quadratically divergent term in the cut-off regularisation can be reabsorbed in the couplings $G_{1,2}$. After the redefinition, the two results are identical if we identify

$$
\frac{1}{\epsilon}-\gamma_{E}+2 \longleftrightarrow \log \frac{\Lambda^{2}}{\pi \mu_{R}^{2}}
$$

However, in both cases the logarithmic divergence cannot be absorbed [36] unless we include extra terms in the Lagrangian like $(\partial \sigma)^{2}$ and $\sigma^{4}$. This is of course a manifestation of the non-renormalizability of the model. For this reason, we shall assume the scale $\Lambda$ (or equivalently $\mu_{R}$ ) to represent a physical cut-off and write

$$
\begin{aligned}
& K_{1}\left(M, \mu, \mu_{5}\right)=\frac{1}{2 \pi^{2}}\left[\Theta ( \mu - M ) \left\{\mu \sqrt{\mu^{2}-M^{2}}\right.\right. \\
& \left.+\left(2 \mu_{5}^{2}-M^{2}\right) \log \left(\frac{\mu+\sqrt{\mu^{2}-M^{2}}}{M}\right)\right\} \\
& \left.-\frac{M^{2}}{2}+\left(M^{2}-2 \mu_{5}^{2}\right) \log \frac{2 \Lambda}{M}\right] .
\end{aligned}
$$

Note that $K_{1}$ increases with $\mu$ and decreases with $\mu_{5}$. The derivative of this function will also be used

$$
\begin{aligned}
& L_{1}\left(M, \mu, \mu_{5}\right) \equiv \frac{1}{M} \frac{\partial K_{1}}{\partial M}=-\frac{1}{\pi^{2}}[\Theta(\mu-M) \\
& \left\{\frac{\mu \mu_{5}^{2}}{M^{2} \sqrt{\mu^{2}-M^{2}}}+\log \left(\frac{\mu+\sqrt{\mu^{2}-M^{2}}}{M}\right)\right\} \\
& \left.+1-\frac{\mu_{5}^{2}}{M^{2}}-\log \frac{2 \Lambda}{M}\right] .
\end{aligned}
$$

It verifies the property $L_{1}\left(\mu_{5}=0\right)>0$.

\section{Search for stable vacuum configurations}

We will now explore the different phases that are allowed by the effective potential (4) by solving the gap equations and analysing the second derivatives to investigate the stable configurations of the different scalar and pseudoscalar condensates. The gap equations for the system read

$$
\begin{aligned}
& \frac{\sigma}{2 G_{1}}+\sum_{ \pm}(M \pm a) K_{1}^{ \pm}=0, \quad \frac{\eta}{2 G_{2}}+\sum_{ \pm}(\eta \pm \Pi) K_{1}^{ \pm}=0, \\
& \frac{\Pi}{2 G_{1}}+\sum_{ \pm} \pm(\eta \pm \Pi) K_{1}^{ \pm}=0, \quad \frac{a}{2 G_{2}}+\sum_{ \pm} \pm(M \pm a) K_{1}^{ \pm}=0
\end{aligned}
$$

where $K_{1}^{ \pm} \equiv K_{1}\left(M_{ \pm}, \mu, \mu_{5}\right)$ (the same convention applies to $L_{1}$ ). The second derivatives of the potential are

$$
\begin{aligned}
& V_{\sigma \sigma}=\frac{1}{2 G_{1}}+\sum_{ \pm}\left[(M \pm a)^{2} L_{1}^{ \pm}+K_{1}^{ \pm}\right], \\
& V_{\eta \eta}=\frac{1}{2 G_{2}}+\sum_{ \pm}\left[(\eta \pm \Pi)^{2} L_{1}^{ \pm}+K_{1}^{ \pm}\right], \\
& V_{\pi \pi}=\frac{1}{2 G_{1}}+\sum_{ \pm}\left[(\eta \pm \Pi)^{2} L_{1}^{ \pm}+K_{1}^{ \pm}\right], \\
& V_{a a}=\frac{1}{2 G_{2}}+\sum_{ \pm}\left[(M \pm a)^{2} L_{1}^{ \pm}+K_{1}^{ \pm}\right], \\
& V_{\sigma \eta}=V_{\pi a}=\sum_{ \pm}(M \pm a)(\eta \pm \Pi) L_{1}^{ \pm}, \\
& V_{\sigma \pi}=V_{\eta a}=\sum_{ \pm} \pm(M \pm a)(\eta \pm \Pi) L_{1}^{ \pm}, \\
& V_{\sigma a}=\sum_{ \pm} \pm\left[(M \pm a)^{2} L_{1}^{ \pm}+K_{1}^{ \pm}\right], \\
& V_{\eta \pi}=\sum_{ \pm} \pm\left[(\eta \pm \Pi)^{2} L_{1}^{ \pm}+K_{1}^{ \pm}\right]
\end{aligned}
$$

To keep the discussion simple we will assume in the subsequent that $a=0$. However, in Sect. 4 we will see that in a very tiny region of the parameter space there is evidence of the existence of a phase with $a \neq 0$. 


\subsection{Chirally symmetric phase}

We will first consider the phase where none of the fields condenses (in the chiral limit with $m=0$ and $\mu=\mu_{5}=0$ for simplicity). The gap equations are automatically satisfied, while the second derivatives read in this case

$V_{\sigma \sigma}=V_{\pi \pi}=\frac{1}{2 G_{1}}+2 K_{1}, \quad V_{\eta \eta}=V_{a a}=\frac{1}{2 G_{2}}+2 K_{1}$,

$V_{\sigma \eta}=V_{\sigma \pi}=V_{\sigma a}=V_{\eta \pi}=V_{\eta a}=V_{\pi a}=0$.

After absorbing the quadratic divergence from the cut-off regularisation into the coupling constants as mentioned previously,

$\frac{1}{2 G_{i}}-\frac{\Lambda^{2}}{\pi^{2}}=\frac{1}{2 G_{i}^{r}}$,

the stability conditions for this phase are $G_{1,2}^{r}>0$. For simplicity, we will drop the superindex $r$ throughout.

\subsection{Chirally broken phase}

In this phase we will explore the phase where the field $\sigma$, and only this field, condenses. The gap equations reduce just to one

$K_{1}=-\frac{1}{4 G_{1}}\left(1-\frac{m}{M}\right)$.

Let us first assume $\mu=\mu_{5}=0$. Then the condition for chiral symmetry breaking (CSB) after absorbing the quadratic divergence into the coupling constants (or right away in DR for that matter) reads

$M^{2}\left(\frac{1}{2}-\log \frac{2 \Lambda}{M}\right)=\frac{\pi^{2}}{2 G_{1}}\left(1-\frac{m}{M}\right)$.

In Fig. 1 we show the region of $G_{1}$ that provides a stable CSB phase with $m=0$ for non-trivial values of the external drivers. All dimensional quantities scale with $\Lambda$, which we take to be $\Lambda=1 \mathrm{GeV}$ throughout. Two discontinuities appear in the plot. The first one is found at

$$
\begin{aligned}
\left(\mu_{5}^{*(1)}\right)^{2}= & \frac{\mu^{2}}{2}\left[\Theta\left(\mu-\mu^{*}\right)\left(1-\frac{1}{2 \ln \frac{2 \Lambda}{\mu}}\right)\right. \\
& \left.+\Theta\left(\mu^{*}-\mu\right) \frac{1}{\ln \frac{\Lambda}{\mu}}\right]
\end{aligned}
$$

with

$$
\begin{aligned}
\mu^{*} \equiv & \exp \left[-\frac{1}{4}\left(3-2 \ln 2+\sqrt{9+4 \ln 2+4 \ln ^{2} 2}\right)\right] \Lambda \\
& \approx 0.265 \Lambda,
\end{aligned}
$$

while the second one can be written analytically only if $\mu<$ $2 \exp \left[-\frac{1}{4}(1+\sqrt{5})\right] \Lambda \approx 0.891 \Lambda$. In this case, the second discontinuity is given by

$$
\left(\mu_{5}^{*(2)}\right)^{2}=(3-\sqrt{5}) \Lambda^{2} \exp \left[-\frac{1}{2}(1+\sqrt{5})\right] \approx(0.389 \Lambda)^{2} .
$$

For $\mu=0$ and $\mu=200 \mathrm{MeV}$, the condition $\mu<0.891 \Lambda$ is satisfied and the previous equation can be used to find the discontinuity, which is clearly independent of $\mu$. The limit $\mu \rightarrow 0$ reduces to $G_{1}<0$, a result known from a previous work on the NJL model in DR [35]. Finally, note that the restriction for $G_{2}$ is simply $\frac{1}{G_{2}}>\frac{1}{G_{1}}$.

The meson spectrum for any value of the external chemical potentials is given by the second derivatives at the local minimum

$$
\begin{aligned}
& V_{\sigma \sigma}=\frac{m}{2 G_{1} M}+2 M^{2} L_{1}, \quad V_{\eta \eta}=\frac{m}{2 G_{1} M}+\frac{1}{2}\left(\frac{1}{G_{2}}-\frac{1}{G_{1}}\right), \\
& V_{\pi \pi}=\frac{m}{2 G_{1} M}, \quad V_{a a}=\frac{m}{2 G_{1} M}+\frac{1}{2}\left(\frac{1}{G_{2}}-\frac{1}{G_{1}}\right)+2 M^{2} L_{1}, \\
& V_{\sigma \eta}=V_{\sigma \pi}=V_{\sigma a}=V_{\eta \pi}=V_{\eta a}=V_{\pi a}=0,
\end{aligned}
$$

where one has to use a bare quark mass $m$ of the same sign as the coupling $G_{1}$ so as to provide a positive pion mass.

The stability conditions read

$$
\frac{1}{G_{2}}>\frac{1}{G_{1}}\left(1-\frac{m}{M}\right), \quad 2 M^{2} L_{1}>\max \left[-V_{\pi \pi},-V_{\eta \eta}\right] .
$$

Fig. 1 Allowed region of $G_{1}$ as a function of $\mu_{5}$ with fixed $\mu$ for a stable CSB phase (dark region). The left panel shows $\mu=0$, while the right one corresponds to $\mu=200 \mathrm{MeV}$. The figure corresponds to $m=0$ and $\Lambda=1 \mathrm{GeV}$
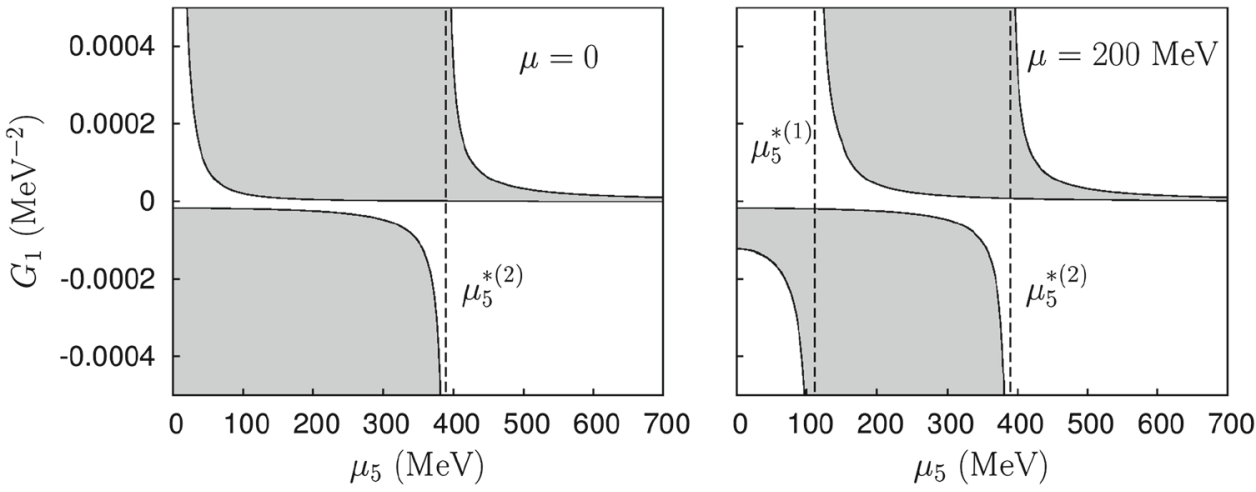
Fig. 2 Evolution of the constituent quark mass $M$ depending on $\mu$. For both plots we set $G_{2}=-45 / \Lambda^{2}$ with $\Lambda=1 \mathrm{GeV}$ and $\mu_{5}=0$. In the left panel, we fixed $G_{1}=-40 / \Lambda^{2}$ and plot for different values of $m$. In the right panel instead, we fixed $m=-5 \mathrm{MeV}$ in order to examine the variation of $G_{1}$. The transition becomes sharper as $m$ decreases
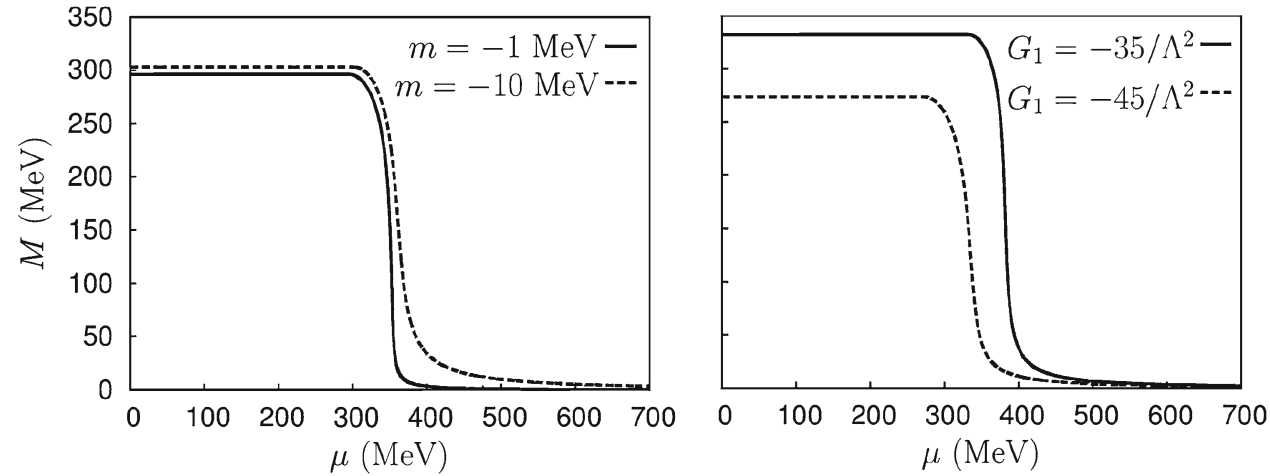

Let us set once again $\mu=\mu_{5}=0$. Then $L_{1}>0$ and the second convexity condition is always met if the first one is fulfilled. In this case the mass spectrum obeys the relation

$m_{\sigma}^{2}-m_{\pi}^{2}=m_{a}^{2}-m_{\eta}^{2}>0$,

in analogy to the situation in QCD. In addition the following relation also holds:

$m_{a}^{2}-m_{\sigma}^{2}=m_{\eta}^{2}-m_{\pi}^{2}$,

and the difference $m_{\eta}^{2}-m_{\pi}^{2}$ is positive (like the analogous one in QCD [37-40] for a review see [41]) provided that $\frac{1}{G_{2}}-\frac{1}{G_{1}}>0$.

Let us now examine in detail the dependence of the chiral condensate on the external chemical potentials. In Fig. 2 we present the evolution of the constituent quark mass as a function of the vector chemical potential for different values of the current quark mass and coupling $G_{1}$ (left and right panels, respectively) with $\mu_{5}=0$. Both the bare quark mass and the coupling $G_{1}$ are taken to be negative, as just explained above. There is chiral restoration around a certain value of the chemical potential that depends mostly on $G_{1}$; this phenomenon of chiral restoration is well known in the NJL model [42] and it is possibly the main reason that this simple model fails to reproduce correctly the transition to nuclear matter. The transition becomes sharper as the value $m=0$ is approached.

In Fig. 3 we observe the influence of the axial chemical potential $\mu_{5}$ on the restoration of chiral symmetry that always takes place in the NJL as $\mu$ increases. For high values of the axial chemical potential, the plateau appearing for $M>\mu$ acquires bigger values and spreads over a wider range of $\mu$. At some point, the solution of the gap equation shows a stable and a metastable solution that must necessarily flip thus implying a jump of the constituent quark mass at some value of the chemical potential where both solutions coexist. Between these solutions, another unstable solution exists, but it is not shown in the plot since the Hessian matrix is not positive definite. The jump represents a first order phase transition from $\mu<M$ (=constant) to a non-constant $M$ smaller than the chemical potential.

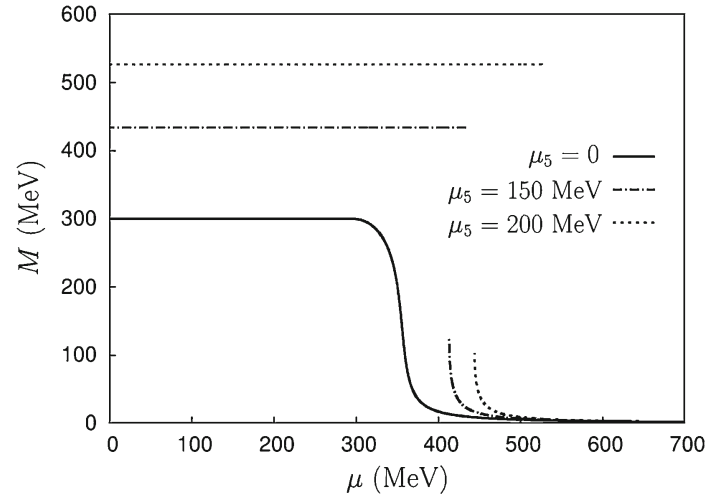

Fig. 3 Evolution of the constituent quark mass $M$ depending on $\mu$ for different values of the axial chemical potential $\mu_{5}$ setting $m=-5 \mathrm{MeV}$, $G_{1}=-40 / \Lambda^{2}$ and $G_{2}=-45 / \Lambda^{2}$. The drawn lines correspond to locally stable phases and accordingly the absence of a continuous line in the cases where $\mu_{5} \neq 0$ is due to the fact that the Hessian matrix is not positive definite. The transition to a chirally restored phase changes to a first order one as $\mu_{5}$ increases

It may be helpful to show a plot of the same constituent quark mass depending on $\mu_{5}$ for different values of $\mu$. In the left panel of Fig. 4 we display such evolution for $\mu=0$ and $390 \mathrm{MeV}$. The first curve is valid for any $\mu<M \approx 300 \mathrm{MeV}$ while the second one shows a small discontinuity that represents a first order phase transition within the CSB phase. A detail of the jump is presented in the inset. Note that both curves coincide after the jump and stop at $\mu_{5} \sim 280 \mathrm{MeV}$ since beyond this value, the phase becomes unstable, as presented previously in Fig. 1.

In the right panel, we present the values of $\mu=395$ and $410 \mathrm{MeV}$, which correspond to qualitatively different cases. The curve for $\mu=395 \mathrm{MeV}$ shows two separate regions where the function is bivaluated. First, the lower and intermediate branches share some common values of $\mu_{5}$ even that it cannot be appreciated in the plot. Thus, a first order phase transition must take place within this region. The same behaviour happens for the intermediate and the upper branches, implying another first order phase transition. For bigger values of $\mu_{5}$ one recovers the tendency of $\mu=0$ as in the previous case. The curve $\mu=410 \mathrm{MeV}$ is somewhat 


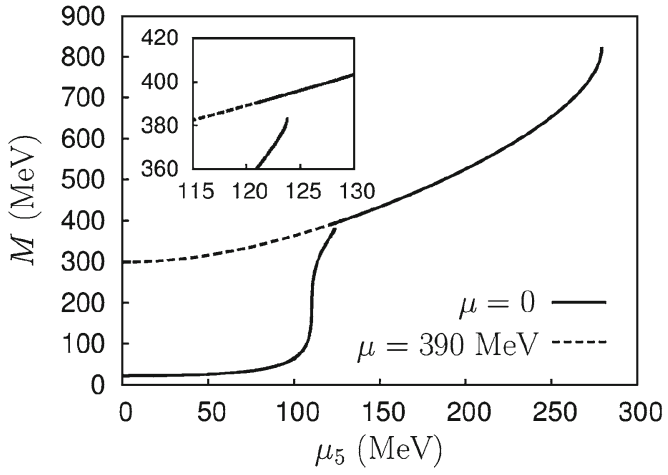

Fig. 4 Evolution of the constituent quark mass $M$ depending on $\mu_{5}$ for different values of the chemical potential $\mu$ setting $m=-5 \mathrm{MeV}$, $G_{1}=-40 / \Lambda^{2}$ and $G_{2}=-45 / \Lambda^{2}$. Both graphics show the regions where all the second derivatives are positive. Certain values of $\mu_{5}$ exhibit coexisting solutions implying first order phase transitions. In the left

similar to the previous one but now with a trivaluated region: for a certain small range of $\mu_{5}$ the three branches may be reached and therefore one or two jumps may take place. For bigger values of $\mu$, the intermediate branch disappears and only one jump may take place.

All the jumps in Fig. 4 are due to the presence of unstable regions that would connect the different branches of the same curve. Here, it can be shown that $V_{\sigma \sigma}<0$ is the responsible for these unstable zones. On the other hand, $V_{a a}$ is simply $V_{\sigma \sigma}$ with a positive shift and the restriction $V_{a a}>0$ does not add anything new.

We want to stress that all the first order phase transitions just explained are a direct consequence of the addition of $\mu_{5}$ to the problem. No other assumptions are made beyond using the mean field approximation.

\section{Isosinglet pseudoscalar condensation and parity breaking}

Next we focus in the analysis of parity-violating phases. It turns out that the only stable one corresponds to condensation in the isoscalar channel. Neutral pseudoscalar isotriplet condensation, either with or without CSB, does not lead to a stable thermodynamical phase ${ }^{2}$. Now, in addition to the scalar condensate $\sigma$, which was explored in the previous section, we will allow for a non-vanishing isosinglet pseudoscalar condensate $\eta$. The gap equations now become

$M=\frac{m}{G_{1}} \frac{1}{\frac{1}{G_{1}}-\frac{1}{G_{2}}}, \quad K_{1}=-\frac{1}{4 G_{2}}$.

2 This is at variance with the QCD- inspired effective theory analysis of $[7,8]$ where the possibility of a condensation in the isotriplet channel was proven.

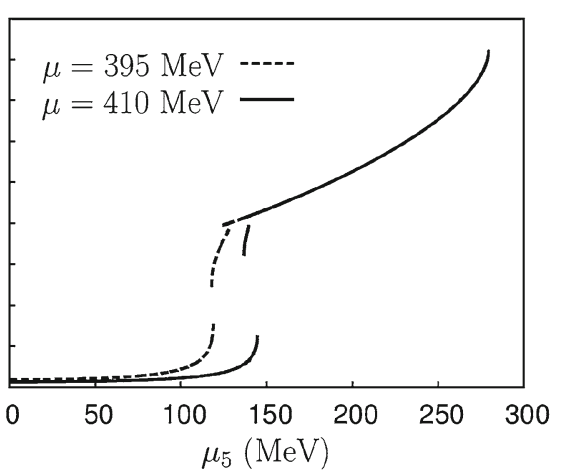

panel, we show a plot for $\mu=0$ (or indeed for any $\mu<M$ ) and $\mu=390 \mathrm{MeV}$. The second curve exhibits a small jump that is shown more detailed in the inset. The right panel corresponds to $\mu=395$ (two jumps) and $410 \mathrm{MeV}$ (probably only one jump). This plot shows that the NJL with external drivers has a rather complex phase diagram

The first gap equation shows that the scalar condensate exhibits a remarkable independence on the external chemical potentials as it turns out to be constant once the parameters of the model are fixed. Unlikely the $\eta$ condensate does depend on the external drivers through the second equation. Moreover, from the first equation one finds that in the paritybreaking phase $m=0$ iff $G_{1}=G_{2}$; namely, the paritybreaking $\eta$ condensate is a stationary point of the effective potential (4) only when the chiral and $U(1)_{A}$ symmetries are explicitly preserved or broken at the same time in the NJL Lagrangian (1). However, this stationary point would not be a true minimum but a stationary point with two flat directions. The more general case where $m \neq 0$ and $G_{1} \neq G_{2}$ is thus the only possibility to have a genuine parity-breaking phase. We will see in a moment how as one takes the limit $m \rightarrow 0$, the narrow window to have access to this phase disappears.

The second derivatives read

$$
\begin{aligned}
V_{\sigma \sigma}= & \frac{1}{2}\left(\frac{1}{G_{1}}-\frac{1}{G_{2}}\right)+2 M^{2} L_{1}, \quad V_{\eta \eta}=2 \eta^{2} L_{1}, \\
& V_{\sigma \eta}=2 M \eta L_{1}, \\
V_{\pi \pi}= & \frac{1}{2}\left(\frac{1}{G_{1}}-\frac{1}{G_{2}}\right)+2 \eta^{2} L_{1}, \quad V_{a a}=2 M^{2} L_{1}, \\
& V_{\pi a}=2 M \eta L_{1}, \\
V_{\sigma \pi}= & V_{\sigma a}=V_{\eta \pi}=V_{\eta a}=0 .
\end{aligned}
$$

We find that the Hessian matrix is not diagonal but has a block structure with two isolated sectors $\sigma-\eta$ and $\pi-a$ that reflect the mixing of states with different parity [7-9]. The determinants of these blocks are

$$
\begin{aligned}
& \operatorname{det}\left(V^{\sigma, \eta}\right)=\eta^{2} L_{1}\left(\frac{1}{G_{1}}-\frac{1}{G_{2}}\right), \\
& \operatorname{det}\left(V^{\pi, a}\right)=M^{2} L_{1}\left(\frac{1}{G_{1}}-\frac{1}{G_{2}}\right),
\end{aligned}
$$


and, thus, the resulting conditions for this phase to be stable reduce to

$L_{1}>0, \quad\left(\frac{1}{G_{1}}-\frac{1}{G_{2}}\right)>0$.

The second of the previous conditions leads to a peculiar ordering of the physical meson spectrum. Recall that in the chiral symmetry breaking phase we had

$m_{a}^{2}-m_{\sigma}^{2}=m_{\eta}^{2}-m_{\pi}^{2}=-\frac{N}{2}\left(\frac{1}{G_{1}}-\frac{1}{G_{2}}\right)$,

and therefore, a stable parity-breaking phase is not compatible with a fit to the phenomenology. Thus parity breaking in the NJL model corresponds to a choice of parameters that makes this model quite different from QCD predictions [3740] for a review see, [41]. In other words, the NJL model with a stable parity-breaking phase will have nothing to do with QCD. Note that the above differences are independent of the phase in which the theory is realised (that is, they are independent of $\mu, \mu_{5}$ ).

The rest of the possible phases with a vanishing $a$ require $m=0$ to satisfy the gap equations; they are not true minima. In particular, there is no phase with parity breaking and $\sigma=0$.

\subsection{Transition to the parity-breaking phase}

In this section we will analyse the characteristics of the transition to the phase where parity is broken. First of all, let us define $M_{0}$ as the solution to $M_{0}=M\left(G_{1}, \mu=\mu_{5}=0\right)$ in the CSB phase given by Eq. (19). Recall the inequality $V_{\eta \eta}>0$ of the same phase given in Eq. (21) and the stability condition of the parity-breaking phase in Eq. (23). Putting all of them together yields the following inequalities:

$0<\frac{1}{G_{1}}-\frac{1}{G_{2}}<\frac{m}{G_{1} M_{0}}$.

The second inequality can be inserted in the first gap equation of the parity-breaking phase (see Eq. (22)) to show that in this phase, $M>M_{0}$. The same set of inequalities can be rewritten as

$\frac{1}{G_{1}}\left(1-\frac{m}{M_{0}}\right)<\frac{1}{G_{2}}<\frac{1}{G_{1}}$,

which means that $G_{1}$ and $G_{2}$ necessarily have the same sign, while in the CSB phase $G_{2}$ had no restriction and could have opposite sign. This set of inequalities represents the necessary condition to have a transition from the CSB to a paritybreaking phase, as they provide the stability conditions of both phases. Notice that the model allows a narrow window of $G_{2}$ (once $G_{1}$ is fixed) so that both phases may take place depending on the value of the external drivers. In the limit $m \rightarrow 0$, this window closes and no parity breaking can be found.
Let us recall the gap equation in the CSB phase Eq. (19) and assume $\mu=\mu_{5}=0$. Provided that Eq. (24) is satisfied, it follows that

$K_{1}=-\frac{1}{4 G_{1}}\left(1-\frac{m}{M_{0}}\right)>-\frac{1}{4 G_{2}}$.

In the parity-breaking phase, the gap equation is $K_{1}=-\frac{1}{4 G_{2}}$; therefore to get into this phase from the familiar CSB one, $K_{1}$ has to decrease, i.e. from (19) we see that $M$ must increase, $M\left(\mu, \mu_{5}\right)>M_{0}$. Let us point out the fact that the condition $L_{1}>0$ from the parity-broken phase is stronger than the one from the CSB one so the former will remain to provide stability to both phases. Let us describe how this process takes place first for $\mu=0$ and finally for $\mu \neq 0$.

\subsection{Phase transition with $\mu=0$}

Let us simplify the analysis by setting $\mu=0$ and let us study the dependence on $\mu_{5}$, which makes $M$ increase from its initial value $M_{0}$. At some critical value such that

$M^{c} \equiv M\left(\mu_{5}^{c}\right)=\frac{m}{G_{1}} \frac{1}{\frac{1}{G_{1}}-\frac{1}{G_{2}}}$,

where the critical value of the axial chemical potential is

$\left(\mu_{5}^{c}\right)^{2}=\frac{M_{c}^{2}}{2}-\frac{1}{4 \log \frac{2 \Lambda}{M_{c}}}\left(M_{c}^{2}-\frac{\pi^{2}}{G_{2}}\right)$,

$m_{\eta}$ vanishes, and from now on we get into the parity-breaking phase via a second order phase transition, where $M$ remains frozen as discussed while the dependence on $\mu_{5}$ is absorbed into a non-vanishing $\eta$ condensate. The dependence of $K_{1}$ on $M_{ \pm}^{2}$ will be now on $M_{c}^{2}+\eta^{2}$. Note that $\left(\mu_{5}^{c}\right)^{2}>0$ and, therefore, a threshold in $M^{c}$ follows.

In Fig. 5 we present a plot showing the evolution of $M$ and $\eta$ with respect to $\mu_{5}$ for $\mu=0$ (or any $\mu<M_{0} \approx$ $300 \mathrm{MeV}$ ). In the CSB phase $M$ grows with $\mu_{5}$ up to the critical value $M^{c}$, the point where this magnitude freezes

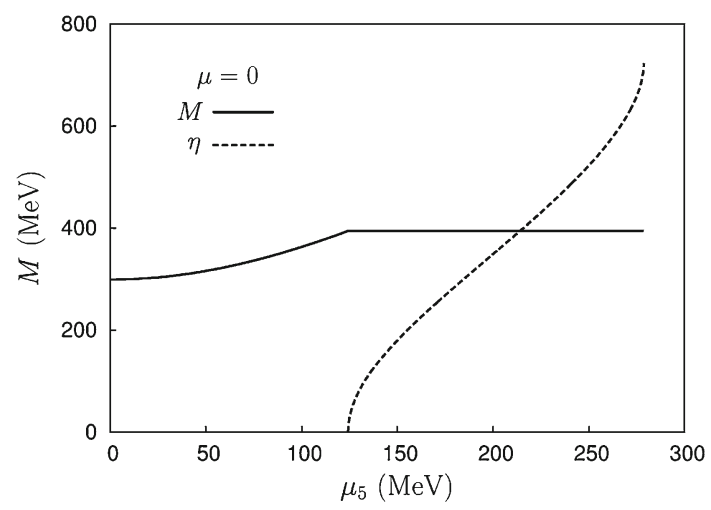

Fig. $5 M$ and $\eta$ dependence on $\mu_{5}$ for $\mu<M_{0}, G_{1}=-40 / \Lambda^{2}$, $G_{2}=-39.5 / \Lambda^{2}, m=-5 \mathrm{MeV}$ and $\Lambda=1 \mathrm{GeV}$ 
Fig. $6 M$ and $\eta$ dependence on $\mu_{5}$ for $\mu=375,390,400$ and $425 \mathrm{MeV}, G_{1}=-40 / \Lambda^{2}$, $G_{2}=-39.5 / \Lambda^{2}, m=-5 \mathrm{MeV}$ and $\Lambda=1 \mathrm{GeV}$. The graphics show the regions where all the second derivatives are positive. Certain values of $\mu_{5}$ exhibit coexisting solutions, implying first order phase transitions. The first jump in the plot for $\mu=390 \mathrm{MeV}$ shows a very small region of $\mu_{5}$ where the function is not defined. This region is characterised by $V_{a a}<0$, thus suggesting a phase with a non-trivial scalar isotriplet condensate. This is the only region where we have found indications for a phase with $a \neq 0$. The landscape of first order phase transitions in the constituent quark mass is essentially the same as the one explained in Fig. 4
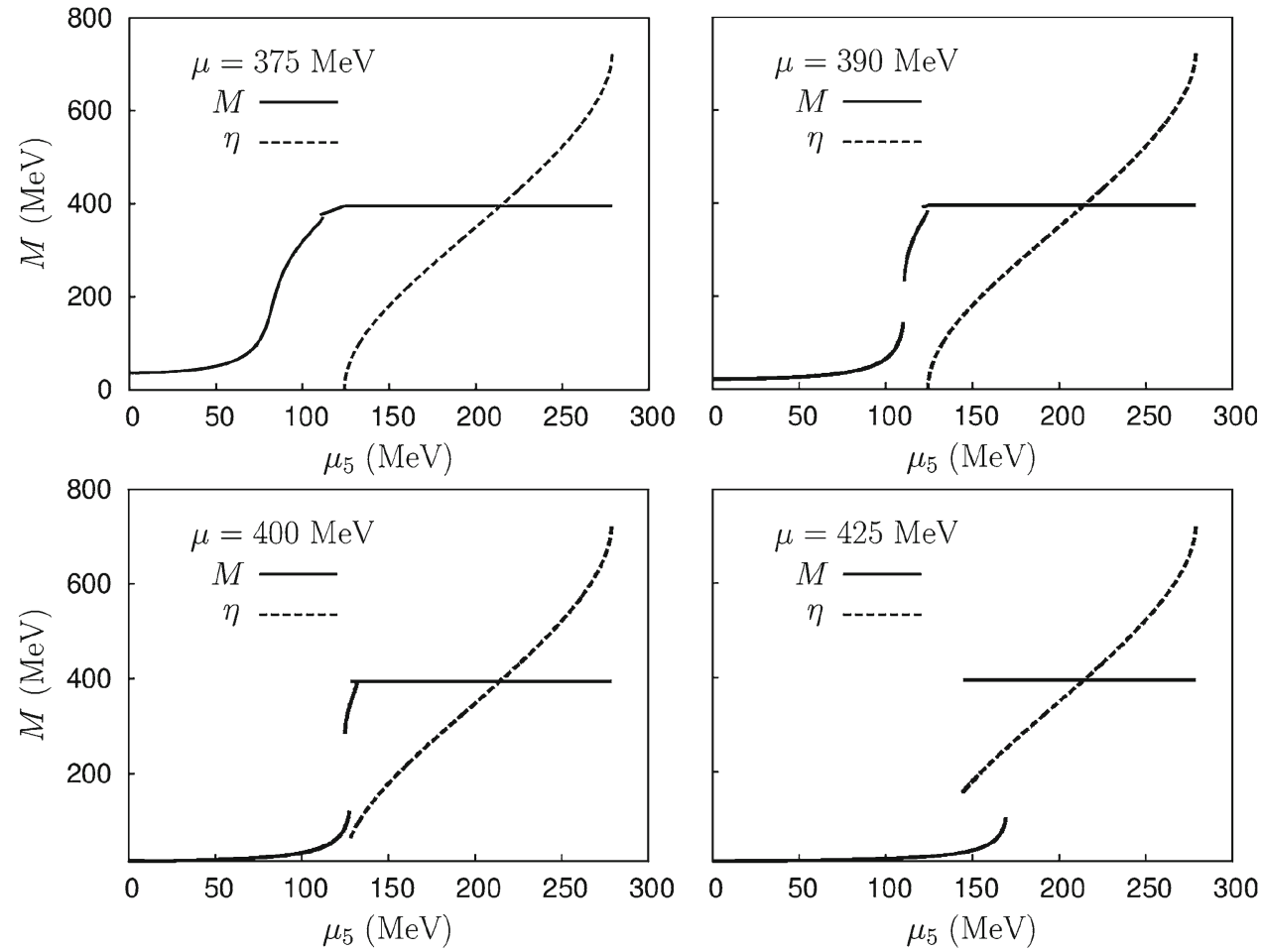

out, and $\eta$ acquires non-trivial values, also growing with the axial chemical potential. At $\mu_{5} \simeq 0.28 \Lambda$, this phase shows an endpoint, and beyond it no stable solution exists. This point is the same one as we found in the CSB phase, meaning that the model becomes unstable at such a value of $\mu_{5}$, no matter which phase one is exploring.

\subsection{Phase transition with $\mu>0$}

The presence of both chemical potentials makes the function $K_{1}$ exhibit more complicated features. As $K_{1}$ decreases with $\mu_{5}$ and $\mu$ does the opposite job, $\mu_{5}$ needs larger values than $\mu$ to reach the parity-breaking phase. In Fig. 6, we present a set of plots with the evolution of both $M$ and $\eta$ for nonvanishing values of the chemical potential. As before we take the value $\Lambda=1 \mathrm{GeV}$ to make the model in order to have some QCD-inspired intuition. Of course everything scales with $\Lambda$.

In the upper panels, we set $\mu=375 \mathrm{MeV}$ (left) and $\mu=$ $390 \mathrm{MeV}$ (right), both of them $M_{0}<\mu<M^{c}$, where jumps in $M$ are observed in the parity even phase together with tiny metastable regions. This behaviour is very similar to the one described in Fig. 4 with the subtlety that we inverted the sign of $\frac{1}{G_{1}}-\frac{1}{G_{2}}$ and therefore, the parity-odd phase may be reached.

In addition, this change of sign shifts the second derivative $V_{a a}$, which is the only responsible for the apparent big jump in the $\mu=390 \mathrm{MeV}$ window (the one with lower $\mu_{5}$ ).
It should be clear that $L_{1}>0$ since $M$ is growing with $\mu_{5}$. However, the second derivative $V_{a a}$ becomes negative due to this shift while all the other derivatives remain positive. If for a moment we forgot $V_{a a}$, the curve would be smoothly increasing and we would only have the other tiny jump close to the flat region of constant $M$. However, the fact that this second derivative becomes negative leads to a small range of $\mu_{5}$ where no solution exists. Hence, it seems natural to think that the system goes away from the phase with $a=0$ and acquires a non-trivial scalar isotriplet condensate. We emphasise that this region is really tiny and depends crucially on the specific values for the parameters, even disappearing for $G_{1}>-30 / \Lambda^{2}$. Both graphics show a smooth transition to the parity-odd phase, say, via a second order phase transition with the same characteristics of the previous section with $\mu=0$.

On the other side, in the lower panels, we set $\mu=$ $400 \mathrm{MeV}$ (left) and $\mu=425 \mathrm{MeV}$ (right) with $\mu>M^{c}$ and observe what we could more or less expect from Fig. 4 with the same landscape of first order phase transitions. The main difference of these two latter values appears in the finite jump of $\eta$, implying now a first order phase transition toward the parity-breaking phase.

Finally, we present the phase transition line in a $\mu^{c}\left(\mu_{5}^{c}\right)$ plot in Fig. 7. For $\mu<M^{c} \approx 395 \mathrm{MeV}$ (or equivalently, for $\left.\mu_{5}^{c}=\mu_{5}^{c}(\mu=0)\right)$, the transition is smooth (second order) while beyond that there is a jump in the condensates (first order), as was also observed in the previous figure. 


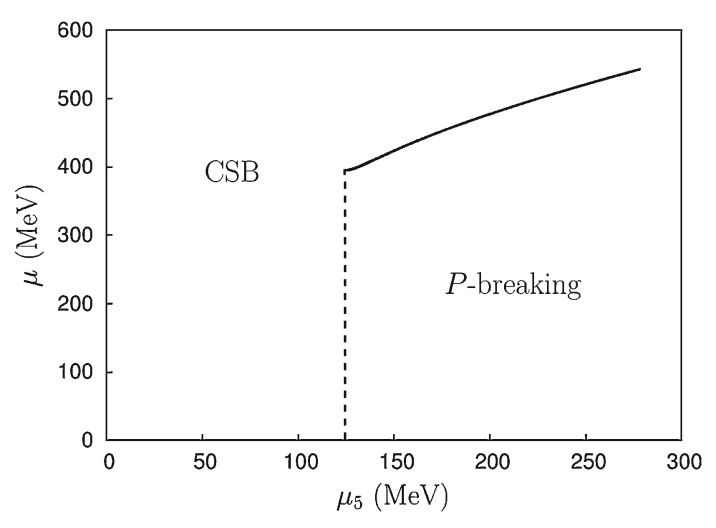

Fig. 7 Transition line from the CSB to the $P$-breaking phase with $G_{1}=-40 / \Lambda^{2}, G_{2}=-39.5 / \Lambda^{2}, m=-5 \mathrm{MeV}$ and $\Lambda=1 \mathrm{GeV}$. The vertical dashed line is related to a second order phase transition while the solid one corresponds to a first order one

\section{Conclusions and outlook}

The Nambu-Jona-Lasinio model has traditionally received much attention as a toy model for QCD. In spite of the obvious shortcomings of this analogy, NJL is regarded as providing an intuitive picture of the mechanism of chiral symmetry breaking in QCD via a strong effective interaction in the scalar isosinglet channel. More recently the NJL model has received attention as a simpler arena where other aspects of QCD could be tested, such as extreme QCD. Although it is far from obvious that NJL is a good modellisation of QCD, these tests are still useful to understand in a simpler theory what are the right questions to pose.

In this context, the NJL model has been used recently by some authors [43] to investigate the nature of the Aoki phase in QCD $[44,45]$. This is a phase in lattice QCD with Wilson fermions where parity and possibly isospin symmetry is broken. It does not survive the continuum (note that the NJL does not have a 'continuum limit' either). It is, however, conceivable that the introduction of the chemical potential may enlarge the scope of the Aoki phase and allow for a sensible continuum interpretation. This is what should happen if the effective theory analysis of some of the present authors described in $[7,8]$ is correct. Finite chemical potential simulations being notoriously difficult in lattice QCD, it is worth to analyse simpler theories such as NJL where analytical methods are available in the large $N$ limit.

The generation of an axial charge in heavy ion collision processes has also been contemplated in the theory. The effects on QCD phenomenology of such a charge have been barely considered in the past. NJL may provide a first guidance to the problem too.

In this paper we work in the continuum and explore in detail the different phases that arise in the Nambu-JonaLasinio model in the presence of both vector and axial chemical potentials at zero temperature. The incorporation of $\mu_{5}$ together with $\mu$ had not been investigated before. The axial chemical potential changes considerably the thermodynamical properties of the model. It leads to a non-trivial dependence of the scalar condensate in the chirally broken phase. Interestingly, when the full $U(2)_{L} \times U(2)_{R}$ global symmetry is broken to $S U(2)_{L} \times S U(2)_{R} \times U(1)_{V}$ (i.e. $\left.G_{1} \neq G_{2}\right)$ a phase where parity is spontaneously broken by the presence of an isosinglet condensate $\eta$ appears. However, we have not found any phase where parity and flavour symmetry are simultaneously broken, thus indicating the presence of a nonzero value for $\left\langle\bar{\psi} \gamma_{5} \tau^{3} \psi\right\rangle$. On the contrary we have found an extremely small region in the $\mu-\mu_{5}$ space of parameters where flavour symmetry is broken by a non-zero value of $\left\langle\bar{\psi} \tau^{3} \psi\right\rangle$ but parity is not broken yet. However, the appearance of a parity-breaking condensate in the isosinglet sector is rather generic for $m \neq 0$.

Demanding stability of such a phase, however, leads to a region of parameter space where the spectrum has little resemblance to the one of QCD. We have investigated all the properties of the transition from the parity-even to the parity-odd phase providing results on the evolution of both condensates, which exhibit finite jumps under certain conditions, and, finally examining the phase transition line, where it was shown that for $\mu<M^{c}$ we have a second order transition while for $\mu>M^{c}$, it corresponds to a first order one.

The discussion presented here on the phase structure of the NJL model in the presence of external chemical potentials is rather general and, as discussed above, the model-in spite of its simplicity - exhibits an enormously rich phase structure. This hopefully indicates that QCD still holds many surprises for us too.

Acknowledgments We would like to thank V. Azcoiti and E. Follana for numerous discussions concerning parity breaking in the NJL model and, particularly, for clarifying to us several points on the reality and positivity properties of the fermion determinant. We acknowledge the financial support from projects FPA2010-20807, 2009SGR502, CPAN (Consolider CSD2007-00042). A. A. Andrianov is also supported by Grant RFBR project 13-02-00127 as well as by the Saint Petersburg State University grant 11.38.660.2013. X. Planells acknowledges the support from Grant FPU AP2009-1855.

Open Access This article is distributed under the terms of the Creative Commons Attribution License which permits any use, distribution, and reproduction in any medium, provided the original author(s) and the source are credited.

Funded by $\mathrm{SCOAP}^{3}$ / License Version CC BY 4.0.

\section{Appendix A: Calculation of the fermion determinant}

In this appendix we address the analysis of the determinant of the fermion operator presented in Eq. (5),

$\mathcal{M}\left(\mu, \mu_{5}\right)=\not \partial+(M+\vec{\tau} \vec{a})-\mu \gamma_{0}-\mu_{5} \gamma_{0} \gamma_{5}+i \gamma_{5}(\vec{\tau} \vec{\tau}+\eta)$. 
As has been already stressed in [43], the fermion determinant can be proven to be real. The presence of both a vector and an axial chemical potential does not modify this feature. Invariance under parity- and time-reversal symmetries also provides some equalities that will be useful for our purposes,

$$
\begin{aligned}
\operatorname{det}\left(\mathcal{M}\left(\mu, \mu_{5}\right)\right) & =\operatorname{det}\left(\mathcal{M}^{\dagger}\left(\mu, \mu_{5}\right)\right)=\operatorname{det}\left(\mathcal{M}\left(\mu,-\mu_{5}\right)\right) \\
& =\operatorname{det}\left(\mathcal{M}^{\dagger}\left(\mu,-\mu_{5}\right)\right) .
\end{aligned}
$$

We shall choose $N$ to be even in order for the determinant to be positive defined and use the fact that $\operatorname{det}(\mathcal{M})^{2}=$ $\operatorname{det}\left(\mathcal{M}^{2}\right)$. The development of the product

$$
\begin{aligned}
& \mathcal{M}\left(\mu, \mu_{5}\right) \mathcal{M}^{\dagger}\left(\mu,-\mu_{5}\right) \\
& =-\partial^{2}+M^{2}+\vec{\pi}^{2}+\left(\eta^{2}+\vec{a}^{2}\right)+2 M \vec{\tau} \vec{a}+2 \eta \vec{\tau} \vec{\pi} \\
& \quad+2 \gamma_{5}(\vec{a} \times \vec{\pi}) \vec{\tau}-\mu^{2}+\mu_{5}^{2}+2 \mu \partial_{0}-2 \mu_{5} \gamma_{0} \vec{\gamma} \vec{\partial} \gamma_{5}
\end{aligned}
$$

provides a result which is scalar in flavour except for the term proportional to $\mu_{5}$. An additional product produces

$$
\begin{aligned}
& \mathcal{M}\left(\mu, \mu_{5}\right) \mathcal{M}^{\dagger}\left(\mu,-\mu_{5}\right) \mathcal{M}\left(\mu,-\mu_{5}\right) \mathcal{M}^{\dagger}\left(\mu, \mu_{5}\right) \\
& =A^{\prime}+\vec{\tau}\left(\vec{\alpha}^{\prime}+\vec{\epsilon}^{\prime} \gamma_{5}\right)
\end{aligned}
$$

with

$$
\begin{aligned}
& A^{\prime}=A^{2}+\vec{\alpha}^{2}+\vec{\epsilon}^{2}+4 \mu_{5}^{2} \vec{\partial}^{2}, \quad \vec{\alpha}^{\prime}=2 A \vec{\alpha}, \quad \overrightarrow{\epsilon^{\prime}}=2 A \vec{\epsilon}, \\
& A=-\partial^{2}+M^{2}+\vec{\pi}^{2}+\left(\eta^{2}+\vec{a}^{2}\right)-\mu^{2}+\mu_{5}^{2}+2 \mu \partial_{0}, \\
& \vec{\alpha}=2(M \vec{a}+\eta \vec{\pi}), \\
& \vec{\epsilon}=2(\vec{a} \times \vec{\pi}), \quad \vec{\alpha} \vec{\epsilon}=0,
\end{aligned}
$$

with the property $\vec{\alpha}^{\prime} \vec{\epsilon}^{\prime}=0$. The logarithm of a quantity with such characteristics can be calculated and all the nondiagonal operators in Dirac or flavour space disappear, leading to

$\log \left[A+\vec{\tau}\left(\vec{\alpha}+\vec{\epsilon} \gamma_{5}\right)\right]=\frac{1}{2} \log \left[A^{2}-\vec{\alpha}^{2}-\vec{\epsilon}^{2}\right]$.

The evaluation of the argument leads to

$$
\begin{aligned}
A^{\prime 2}-\vec{\alpha}^{\prime 2}-\vec{\epsilon}^{\prime 2}= & \prod_{ \pm}\left[-\left(i k_{0}+\mu\right)^{2}+\left(|\vec{k}| \pm \mu_{5}\right)^{2}+M_{+}^{2}\right] \\
& {\left[-\left(i k_{0}+\mu\right)^{2}+\left(|\vec{k}| \pm \mu_{5}\right)^{2}+M_{-}^{2}\right] }
\end{aligned}
$$

where $M_{ \pm}^{2}=(M \pm a)^{2}+(\eta \pm \pi)^{2}$. Finally the fermion determinant can be written as

$$
\begin{aligned}
\log \operatorname{det}\left(\mathcal{M}\left(\mu, \mu_{5}\right)\right) \\
=\operatorname{Tr} \log \mathcal{M}\left(\mu, \mu_{5}\right)=\frac{1}{8} \operatorname{Tr} \log \left(A^{\prime 2}-\vec{\alpha}^{\prime 2}-{\overrightarrow{\epsilon^{\prime}}}^{2}\right) \\
=\frac{1}{8} \operatorname{Tr} \sum_{ \pm}\left\{\log \left[-\left(i k_{0}+\mu\right)^{2}+\left(|\vec{k}| \pm \mu_{5}\right)^{2}+M_{+}^{2}\right]\right. \\
\left.\quad+\log \left[-\left(i k_{0}+\mu\right)^{2}+\left(|\vec{k}| \pm \mu_{5}\right)^{2}+M_{-}^{2}\right]\right\},
\end{aligned}
$$

where the trace operator is given by

$\operatorname{Tr}(1)=8 N T \sum_{n} \int \frac{d^{3} \vec{k}}{(2 \pi)^{3}}\left[k_{0} \rightarrow \omega_{n}^{F}\right]$,

with $\omega_{n}^{F}=(2 n+1) \pi / \beta$.

\section{References}

1. D. Kharzeev, R.D. Pisarski, M.H.G. Tytgat, Phys. Rev. Lett. 81, 512 (1998)

2. D. Kharzeev, Phys. Lett. B 633, 260 (2006)

3. D. Kharzeev, Ann. Phys. (NY) 325, 205 (2010)

4. D.E. Kharzeev, L.D. McLerran, H.J. Warringa, Nucl. Phys. A 803, $227(2008)$

5. K. Fukushima, D.E. Kharzeev, H.J. Warringa, Phys. Rev. D 78, 074033 (2008)

6. K. Fukushima, D.E. Kharzeev, H.J. Warringa, Nucl. Phys. A 836, $311(2010)$

7. A.A. Andrianov, D. Espriu, Phys. Lett. B 663, 450 (2008)

8. A.A. Andrianov, V.A. Andrianov, D. Espriu, Phys. Lett. B 678, 416 (2009)

9. A.A. Andrianov, D. Espriu, X. Planells, Eur. Phys. J C73, 2294 (2013)

10. P.V. Buividovich, M.N. Chernodub, E.V. Luschevskaya, M.I. Polikarpov, Phys. Rev. D 80, 054503 (2009)

11. P.V. Buividovich, M.N. Chernodub, D.E. Kharzeev, T. Kalaydzhyan, E.V. Luschevskaya, M.I. Polikarpov, Phys. Rev. Lett. 105, 132001 (2010)

12. M. Abramczyk, T. Blum, G. Petropoulos, R. Zhou, PoS LAT 2009, 181 (2009). [arXiv:hep-lat/0911.1348]

13. P.V. Buividovich, T. Kalaydzhyan, M.I. Polikarpov, [arXiv:hep-lat/ 1111.6733v2]

14. M.P. Lombardo, PoS CPOD 2006 (2006). [arXiv:hep-lat/0612017]

15. M.A. Stephanov, PoS LAT2006 (2006) 024. [arXiv:hep-lat/ 0701002]

16. D.T. Son, M.A. Stephanov, Phys. Rev. Lett. 86, 592 (2001)

17. D.T. Son, M.A. Stephanov, Phys. Atom. Nucl. 64, 834 (2001)

18. K. Splittorff, D.T. Son, M.A. Stephanov, Phys. Rev. D 64, 016003 (2001)

19. J.B. Kogut, D. Toublan, Phys. Rev. D 64, 034007 (2001)

20. A. Yamamoto, Phys. Rev. D 84, 114504 (2011)

21. A. Yamamoto, Phys. Rev. Lett. 107, 031601 (2011)

22. M.N. Chernodub, A.S. Nedelin, Phys. Rev. D 83, 105008 (2011)

23. L.-J. Luo, S. Shi, H.-S. Zong, Mod. Phys. Lett. A 28, 1350105 (2013)

24. Y. Nambu, G. Jona-Lasinio, Phys. Rev. 122, 345-358 (1961)

25. Y. Nambu, G. Jona-Lasinio, Phys. Rev. 124, 246-254 (1961)

26. U. Vogl, W. Weise, Prog. Part. Nucl. Phys. 27, 195 (1991)

27. S.P. Klevansky, Rev. Mod. Phys. 64, 649 (1992)

28. T. Hatsuda, T. Kunihiro, Phys. Rep. 247, 221 (1994)

29. M. Buballa, Phys. Rep. 407, 205 (2005)

30. D. Ebert, K.G. Klimenko, Eur. Phys. J C 46, 771-776 (2006)

31. T. Inagaki, D. Kimura, H. Kohyama, A. Kvinikhidze, Phys. Rev. D 85, 076002 (2012)

32. T. Fujihara, T. Inagaki, D. Kimura, A. Kvinikhidze, Prog. Theor. Phys. Suppl. 174, 72 (2008)

33. T. Fujihara, T. Inagaki, D. Kimura, A. Kvinikhidze, Phys. Rev. D 79, 096008 (2009)

34. T. Inagaki, D. Kimura, H. Kohyama, A. Kvinikhidze, Phys. Rev. D 83, 034005 (2011)

35. T. Inagaki, D. Kimura, A. Kvinikhidze, Phys. Rev. D 77, 116004 (2008) 
36. J. Zinn-Justin, Nucl. Phys. B 367, 105 (1991)

37. D. Weingarten, Phys. Rev. Lett. 51, 1830 (1983)

38. C. Vafa, E. Witten, Phys. Rev. Lett. 53, 535 (1984)

39. S. Nussinov, Phys. Rev. Lett. 52, 966 (1984)

40. D. Espriu, M. Gross, J.F. Wheater, Phys. Lett. B 146, 67 (1984)

41. S. Nussinov, M. Lambert, Phys. Rep. 362, 193 (2002)
42. M. Buballa, Nucl. Phys. A 611, 393-408 (1996)

43. V. Azcoiti, G. Di Carlo, E. Follana, M. Giordano, A. Vaquero, Nucl. Phys. B 875, 45-62 (2013)

44. S. Aoki, Phys. Rev. D 30, 2653 (1984)

45. S. Aoki, Phys. Rev. Lett. 57, 3136 (1986) 